\title{
Paramètres de population et taux d'exploitation de Sarotherodon melanotheron melanotheron Rüppell (1852, Cichlidae) et Ethmalosa fimbriata (Bowdich, 1825, Clupeidae) dans le lac Ahémé et ses chenaux avant le dragage (Bénin, Afrique de l'Ouest)
}

\author{
C.C. VIAHO ${ }^{1,3^{*}}$, D. LEDEROUN ${ }^{1}$, I. S. BAGLO ${ }^{1}$, S. AHOUANSSOU MONTCHO ${ }^{2}$, \\ D. ADANDEDJAN ${ }^{1}$, T. AGBLONON HOUELOME ${ }^{1}$, N.M. GBEDEY ${ }^{3}$ et P. LALEYE ${ }^{1}$ \\ ${ }^{1}$ Laboratoire d'Hydrobiologie et d'Aquaculture, Faculté des sciences agronomiques, Université d'Abomey- \\ Calavi, 01 BP : 526 Cotonou, Bénin. \\ ${ }^{2}$ Laboratoire de Recherche en Aquaculture et en Biologie et Ecologie Aquatiques, Ecole d'Aquaculture de \\ Vallée, Université Nationale d'Agriculture (UNA), Bénin. \\ ${ }^{3}$ Agence pour le Développement intégré de la zone Economique du Lac Ahémé et ses Chenaux (ADELAC). \\ Sègbohouè, Kpomassè, Bénin. BP : 01 SEGBOHOUE. \\ *Auteur correspondant ; E-mail: viahochristian@gmail.com
}

\author{
Received: 15-06-2021 \\ Accepted: $20-10-2021$ \\ Published: 30-10-2021
}

\section{RESUME}

Sarotherodon melanotheron melanotheron Rüppell (1852, Cichlidae) et Ethmalosa fimbriata (Bowdich, 1825, Clupeidae) figurent parmi les espèces de poisson les plus exploitées et les plus consommées par les populations riveraines du lac Ahémé et ses chenaux. En vue de réévaluer les paramètres de la population et le niveau d'exploitation de $S$. m. melanotheron et donner pour la première fois l'état des lieux pour E. fimbriata, la présente étude a été initiée. Les échantillons ont été collectés à partir des captures des pêcheurs d'octobre 2016 à septembre 2017. Les paramètres calculés sont: la relation poids-longueur, la taille de première maturité sexuelle, la mortalité par pêche, le taux d'exploitation, la taille de première capture, la longueur optimale et le recrutement saisonnier du poisson. Sur 1558 spécimens de Ethmalosa fimbriata analysés, la longueur totale a varié de 4,9 à 10,8 cm et le poids de 1,7 à 46,24 g, tandis que sur 2882 spécimens de $S$. m. melanotheron examinés, la longueur totale a varié de 5,8 à 18,4 cm et le poids total a varié de 3,4 à 109,77 g. Le coefficient d'allométrie $\mathrm{b}$ a été significativement différent de 3 pour les deux espèces et la croissance a été de type allométrie négative pour $S$. m. melanotheron et de type allométrie positive $E$. fimbriata. La taille de première maturité sexuelle a été estimée à 12,20 et $10,30 \mathrm{~cm}$ respectivement pour les mâles et les femelles de $S$. m. melanotheron et 8,3 et 7,5 cm respectivement pour les mâles et les femelles de E. fimbriata. La mortalité totale a été estimée à 2,56 et 2,89 $\mathrm{an}^{-1}$ respectivement pour $S$. m. melanotheron et E. fimbriata. Le recrutement est étendu sur toute l'année chez les deux espèces. Les caractéristiques environnementales, les paramètres physico-chimiques du milieu, la pression de pêche ainsi que le degré du parasitisme et toutes autres activités anthropiques sur le milieu sont autant de facteurs dont leurs variations ont influencé la croissance des espèces de poissons qui y vivent. Le stock de $S$. m. melanotheron dans le lac Ahémé et ses chenaux peut être supposé surexploité alors que celui de E. fimbriata reste non surexploité.

(C) 2021 International Formulae Group. All rights reserved.

Mots clés : Surexploitation, mortalité par pêche, lac Ahémé et ses chenaux, sensibilisation. 


\title{
Population parameters and exploitation rate of Sarotherodonmelanotheronmelanotheron Rüppell (1852, Cichlidae) and Ethmalosafimbriata (Bowdich, 1825, Clupeidae) in Lake Ahémé and its channels before dredging (Benin, West Africa)
}

\begin{abstract}
Sarotherodon melanotheron melanotheron Rüppell (1852, Cichlidae) and Ethmalosa fimbriata (Bowdich, 1825, Clupeidae) are among the most exploited fish species and the most consumed by the riparian populations of Ahémé Lake and its channels. In order to reassess the population parameters the and the exploitation level of $S$. m. melanotheron and provide for the first time the status report for E. fimbriata, the present study has been initiated. The samples were collected from the catches of fishermen from October 2016 to September 2017. The parameters estimated were: the weight-length relationship, the size at first sexual maturity, the fishing mortality, the exploitation rate, the size catch, the optimal length and seasonal recruitment of fish. Out of 1558 specimens of Ethmalosa fimbriata analyzed, the total length varied from 4.9 to $10.8 \mathrm{~cm}$ and the weight from 1.7 to $46.24 \mathrm{~g}$, while on 2882 specimens of $S$. m. melanotheron examined, the total length varied from 5.8 to $18.4 \mathrm{~cm}$ and the total weight varied from 3.4 to $109.77 \mathrm{~g}$. The allometric coefficient b was significantly different from 3 for the two species and the growth was negative allometric for $S$. m. melanotheron and E. fimbriata positive allometric type. The size at first sexual maturity was estimated at 12.20 and $10.30 \mathrm{~cm}$ respectively for males and females of $S$. m. melanotheron and 8.3 and $7.5 \mathrm{~cm}$ respectively for males and females of E. fimbriata. Total mortality was estimated at 2.56 and $2.89 \mathrm{yr}-1$ respectively for $S$. $m$. melanotheron and $E$. fimbriata. Recruitment is spread throughout the year in both species. The environmental characteristics, the physicochemical parameters of the environment, the fishing pressure as well as the degree of parasitism and all other anthropogenic activities on the environment are all factors whose variations have influenced the growth of the fish species that live there. The stock of $S$. m. melanotheron in lake Ahémé and its channels may be assumed to be overexploited, while that of $E$. fimbriata remains unexploited

(C) 2021 International Formulae Group. All rights reserved.
\end{abstract}

Keywords: Overexploitation, fishing mortality, Lake Ahémé and its channels, awareness.

\section{INTRODUCTION}

Le Complexe Couffo - lac Ahémé chenal Ahô - lagunes côtières (de Grand-Popo et de Ouidah) est l'un des plus grands plans d'eau du Sud-Ouest Bénin. Il a été déclaré zone humide d'importance internationale (Site Ramsar $N^{\circ} 1017$ ) et contribue à plus de $40 \%$ à la production halieutique continentale au niveau national (Amoussou, 2014). Dans la zone du lac Ahémé et ses chenaux, les exigences de développement socioéconomique ont engendré des activités qui constituent des menaces récursives pour les écosystèmes aquatiques (Villanueva, 2004). Les rejets de déchets industriels et domestiques, le lessivage des terres cultivées et les mauvaises pratiques de pêche fragilisent davantage ces milieux déjà confinés, comblés et caractérisés par un faible renouvellement des eaux (Adandédjan, 2012). Cette situation a entrainé la réduction considérable des captures des produits halieutiques, la précarité des riverains et leur migration vers les pays de la sous-région à la recherche de zones de pêche plus favorables (Viaho, 2014). Face à cette situation, et au regard de l'importance socioéconomique de ces plans d'eau, le Gouvernement du Bénin a décidé de leur réhabilitation à travers la mise en œuvre du "Programme Intercommunal de Réhabilitation du lac Ahémé et ses chenaux et de création d'une zone de développement économique (PIRA)'.' La principale activité de ce programme est le dragage. Cependant, le dragage est une action qui engendre des perturbations écologiques (cas du lac SaintPierre au Canada). Dans ce contexte d'intenses pressions sur ces plans d'eau et dans la perspective du démarrage imminent des opérations de dragage, il est indispensable de 
faire un état des lieux de l'exploitation des espèces de poissons les plus abondantes et les plus exploitées de ces écosystèmes. En effet, les captures au niveau du lac Ahémé et ses chenaux sont dominées par les Cichlidae dont Sarotherodon melanotheron melanotheron Rüppell (1852) représente l'espèce la plus abondante (Lalèyè \& Akélé, 2005; Viaho, 2014). L'espèce Ethmalosa fimbriata (Bowdich, 1825, Clupeidae) y est aussi présente à une proportion non négligeable (4 à 10\% selon les mois) (Niyonkuru, 2007 ; Viaho, 2014). Etant des ressources à valeur économique considérable, la connaissance de la dynamique de ces espèces de poisson est nécessaire pour toute gestion rationnelle des ressources (Al-Barwani et al., 2007 ; Lederoun et al., 2015). Par ailleurs, des études similaires avaient été menées dans plusieurs écosystèmes du pays, mais aucun n'avait pris en compte l'ensemble "lac Ahémé et ses chenaux". La présente étude vient donc combler ce vide en fournissant des données sur la croissance et l'exploitation de ces 02 espèces avant les opérations de dragage. L'objectif de l'étude était d'apporter des informations scientifiques essentielles pour une gestion durable et une exploitation rationnelle de $S$. m. melanotheron et de E. fimbriata. Elle contribuera à réévaluer les paramètres de la population et le niveau d'exploitation de $S$. m. melanotheron et réaliser pour la première fois l'état des lieux pour $E$. fimbriatadans le lac Ahémé et ses chenaux avant le dragage.

\section{MATERIEL ET METHODES Milieu d'étude}

La zone d'étude regroupe le lac Ahémé, le chenal Ahô, la lagune côtière de GrandPopo, la lagune côtière de Ouidah et le canal de Tihimey (Figure 1). Le lac Ahémé reçoit les eaux du fleuve Couffo et se jette dans le chenal Ahô et le canal de Tihimey. Le chenal Ahô se jette dans les lagunes côtières de Grand-Popo et de Ouidah (Amoussou, 2014). La lagune côtière de Grand-Popo, reçoit les eaux du fleuve Mono et se jette dans la mer par l'embouchure "LA BOUCHE DU ROY'. La lagune côtière de Ouidah, quant à elle, prend sa source de la lagune de Grand-Popo et du chenal
Ahô. La présence de l'embouchure fait de la zone d'étude un milieu estuarien lagunaire très diversifié. Le milieu d'étude appartient à la zone subéquatoriale (Sud Bénin) où la pluviométrie varie de $800 \mathrm{~mm}$ à $1200 \mathrm{~mm}$ par an. Il est caractérisé par l'alternance de deux saisons pluvieuses (avril-juillet et octobrenovembre) et de deux saisons sèches (décembre-mars et août-septembre).

\section{Collecte des données}

Les échantillons ont été collectés à partir des captures commerciales entre octobre 2016 et septembre 2017 et identifiés à l'aide de la clé de Teugels \& Thys van den Audenaerde (2003) pour S. m. melanotheron et Gourène et Teugels (2003) pour E. fimbriata. La longueur totale de tous les poissons a été mesurée au centimètre près à l'aide d'un ichtyomètre. Ils ont également été pesés individuellement à l'aide d'une balance électronique de précision $(0,01 \mathrm{~g})$ et sexés (immatures, mâles ou femelles) selon Brown-Peterson et al. (2011).

\section{Traitement des données \\ Relation poids-longueur}

La relation poids-longueur a été établie à partir de l'équation : $\mathrm{Pt}=\mathrm{aLt}^{\mathrm{b}}(\mathrm{Le}$ Cren, 1951 in Lederoun et al., 2005), où Pt est le poids corporel en gramme (g), Lt est la longueur totale en centimètre $(\mathrm{cm})$, a est l'ordonnée à l'origine et $b$ est la pente ou le coefficient de régression ou encore le coefficient d'allométrie. Le coefficient b varie entre 2 et 4 , mais il est souvent proche de 3 . La limite de confiance à $95 \%$ pour b a été évaluée avec le logiciel Statview (Version 1992-98 SAS Institute INC). Afin de vérifier si la valeur de b est significativement différente de 3 , le texte $t$ de Student a été réalisé tel qu'il est exprimé par l'équation selon Sokal \& Rohlf (1987) : ts $=(\mathrm{b}-$ 3) / SE, où ts est la valeur de t-test, b la pente et SE l'erreur-type de la pente b. Le texte est considéré comme significatif au seuil de 5\% (p $<0,05)$.

\section{Taille de première maturité}

La taille de première maturité sexuelle $\mathrm{L}_{50}$ est définie dans la plupart des études comme celle à laquelle 50\% des individus sont 
matures. Dans cette étude, elle a été déterminée aussi bien pour les mâles que pour les femelles à partir de l'équation de la courbe sigmoïde d'évolution des pourcentages $(\mathrm{P})$ de la maturité sexuelle en fonction des classes de taille $(\mathrm{Lt})$.

La courbe sigmoïde calculée d'après le modèle logistique est donnée par la formule suivante (Dagnelie, 1973) :

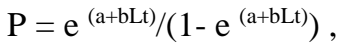

où $\mathrm{a}$ et $\mathrm{b}$ sont des paramètres du modèle. La transformation logarithmique de l'équation permet de la mettre sous la forme $: \ln [\mathrm{P} /(1-\mathrm{P})]$ $=\mathrm{a}+$ bLt et, en remplaçant $\mathrm{P}=50 \%$ dans l'équation, la $\mathrm{L}_{50}$ est obtenue par la formule : $\mathrm{L}_{50}=-\mathrm{a} / \mathrm{b}$. Le logiciel Statview (Version 199298 SAS Institute INC) a été utilisé pour calculer la proportion observée de poissons matures, la proportion estimée et les coefficients $a$ et $b$ du modèle. Les individus aux stades 2 à 5 de maturité sont considérés comme ayant des gonades «actives».

\section{Paramètres de croissance}

A partir des fréquences de longueur totale obtenues par mois, des classes de taille avec un intervalle de $1 \mathrm{~cm}$ ont été établies pour l'étude de la croissance. Le modèle de Von Bertalanffy (1948) a été utilisé à cet effet. Il exprime la longueur Lt du poisson comme fonction de son âge selon l'expression suivante:

$\mathrm{Lt}=\mathrm{L} \infty\left(1-\mathrm{e}^{-\mathrm{K}(\mathrm{t}-\mathrm{to})}\right)$,

Où $\mathrm{Lt}$ : longueur du poisson à l'instant $\mathrm{t} ; \mathrm{L} \infty$ : longueur asymptotique qui serait atteinte par le poisson à l'âge théorique infini ; K: coefficient de croissance caractérisant la vitesse avec laquelle l'espèce croit vers sa taille asymptotique et to : l'âge théorique pour lequel la taille est nulle.

Le programme ELEFAN I (Electronic Length Frequency Analysis) inclus dans FiSAT II (FAO ICLARM Stock Assessment Tools) (Gayalino et al., 1996 in Lederoun et al., 2015) a été mis en œuvre pour évaluer les paramètres $\mathrm{L} \infty$ et $\mathrm{K}$ de l'équation de von Bertalanffy (1957 in Lederoun et al., 2015). Les valeurs $\mathrm{a}$ et $\mathrm{b}$ de la relation poids longueur ont été utilisées.

Les estimations de $\mathrm{L} \infty$ et $\mathrm{K}$ ont permis d'évaluer l'indice de performance de croissance (Pauly et Munro 1984) en utilisant l'équation : $\varphi^{\prime}=\log _{10} \mathrm{~K}+2 \times \log _{10} \mathrm{~L} \infty$.

L'âge théorique to a été obtenu par l'équation de Pauly (1979) :

$\log 10$ (-to) $=-0,392-0,275 \log 10 \mathrm{~L} \infty$ $-1,038 \log 10 \mathrm{~K}$.

La longévité potentielle, tmax, a été calculée par la formule : tmax $=3 / \mathrm{K}$ (Taylor, 1962 ; Pauly, 1980).

\section{Mortalité et taux d'exploitation}

La mortalité totale $\mathrm{Z}$ a été évaluée au moyen du logiciel FiSAT II par la méthode dite « des courbes de captures selon les longueurs converties » à travers la formule $\mathrm{Ln}(\mathrm{Ni} / \mathrm{dti})$. $\mathrm{Ni}$ représente le nombre d'individus dans la classe de taille $\mathrm{i}$ et dt est le temps mis par le poisson pour croître dans cette classe i (Pauly, 1983, 1984, 1990 ; Pauly et al., 1995). La mortalité naturelle $\mathrm{M}$ a été estimée par la méthode de Pauly (1980) basée sur la relation :

$\log 10 \mathrm{M}=0,654 \log 10 \mathrm{~K}-0,28 \log 10 \mathrm{~L} \infty+$ $\log 10 \mathrm{~T}^{\circ} \mathrm{C} \times 0,4634-0,0066$; $\mathrm{K}$ et $\mathrm{L} \infty$ sont les paramètres de l'équation de Von Bertalanffy et $\mathrm{T}$, la température moyenne annuelle du milieu étudié. La température moyenne considérée au cours de cette étude est de $28^{\circ} \mathrm{C}$. La mortalité par pêche $\mathrm{F}$ a été évaluée en utilisant la relation: $\mathrm{F}=\mathrm{Z}-\mathrm{M}$; le taux d'exploitation $\mathrm{E}$ a été obtenu à partir de la relation $: \mathrm{E}=\mathrm{F} / \mathrm{Z}$.

\section{Taille de première capture et taille optimale}

La taille de première capture Lca été évaluée à partir de la procédure de la sélection ogive qui tient compte de la probabilité de capture calculée par chaque classe de taille. La longueur optimale $\mathrm{L}_{\mathrm{opt}}$, pour une cohorte donnée, est estimée selon l'équation (Fröese, 2004) :

$\mathrm{L}_{\mathrm{opt}}=\mathrm{L} \infty \frac{3}{3+\frac{M}{K}} ;$ où $\mathrm{L} \infty$ et $\mathrm{K}$ sont des paramètres de la fonction de croissance de von Bertalanffy et $\mathrm{M}$ est le taux de mortalité naturelle.

\section{Rendement relatif par recrue $Y$ '/R et des points de référence}

Le modèle de Beverton et Holt (1966 in Lederoun et al., 2015), tel que modifié par Pauly et Soriano (1986 in Lederoun et al., 2015), a été utilisé pour prédire le rendement relatif par recrue $\left(\mathrm{Y}^{\prime} / \mathrm{R}\right)$ suivant l'équation : 
$\mathrm{Y}^{\prime} / \mathrm{R}=\mathrm{EU}^{\mathrm{M} / \mathrm{K}}\left[1-(3 \mathrm{U}) /(1+\mathrm{m})+\left(3 \mathrm{U}^{2}\right) /(1+\right.$ $2 \mathrm{~m})-(\mathrm{U} 3) /(1+3 \mathrm{~m})]$

où, $\mathrm{E}=\mathrm{F} / \mathrm{Z}=$ taux d'exploitation actuelle, $\mathrm{Z}$, $\mathrm{M}$ et $\mathrm{F}$ sont respectivement la mortalité totale, naturelle et par pêche,

$\mathrm{U}=1-(\mathrm{Lc} / \mathrm{L} \infty)=$ la fraction de croissance à être achevée par le poisson après son entrée dans la phase d'exploitation $(\mathrm{Lc}=$ longueur moyenne à la première capture, $\mathrm{L} \infty=$ longueur asymptotique),

$\mathrm{m}=(1-\mathrm{E}) /(\mathrm{M} / \mathrm{K})=\mathrm{K} / \mathrm{Z}, \mathrm{K}=$ biomasse relative par recrue $\left(\mathrm{B}^{\prime} / \mathrm{R}\right)$ a été estimé comme étant $\mathrm{B}^{\prime} / \mathrm{R}=\left(\mathrm{Y}^{\prime} / \mathrm{R}\right) / \mathrm{F}$.

D'autres paramètres sont exprimés graphiquement dans ce modèle comme Emax (Exploitation avec rendement productif maximum), E0,1 (Taux d'exploitation pour une augmentation de $\mathrm{Y}^{\prime} / \mathrm{R}$ de $1 / 10$ ème par rapport à $\mathrm{E}=0$ ) et $\mathrm{E} 0,5$ (Valeur de $\mathrm{E}$ sous laquelle le stock a été réduit de $50 \%$ de sa biomasse inexploitée).

\section{Recrutement}

Le modèle de recrutement saisonnier du poisson a été obtenu en utilisant les données de fréquences de longueur restructurées. Cela implique la projection en arrière, le long d'une trajectoire décrite par la courbe de croissance de von Bertalanffy de toutes les données de fréquences de longueur restructurées sur une échelle de temps d'un an (Pauly, 1987). Ainsi, en utilisant ensuite la méthode de probabilité maximale, la distribution est résolue dans ses composantes gaussiennes en utilisant la procédure du « NORMSEP » (séparation normale) de Hasselblad (1966 in Lederoun et al., 2015). Le modèle de recrutement saisonnier a été obtenu en projetant les données de fréquence de longueur en arrière sur une échelle de temps d'un an en utilisant les paramètres de croissance (Dongo et al., 2010).

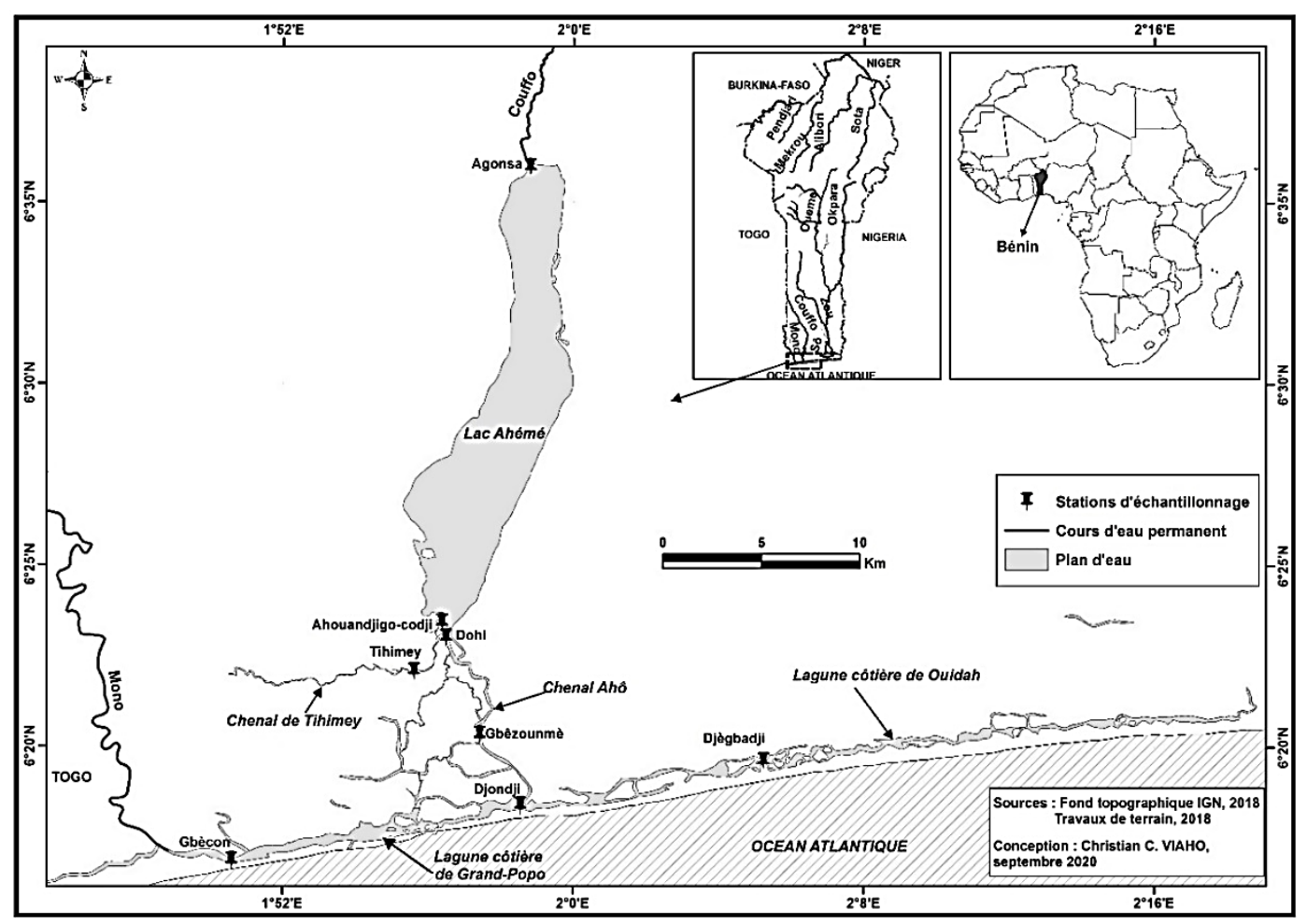

Figure 1: Situation géographique de la zone d'étude. 


\section{RÉSULTATS}

\section{Relation poids-longueur}

Les mesures de longueur et de poids de tous les spécimens de $S$. m. melanotheron $(\mathrm{N}=$ $1558)$ et Ethmalosa fimbriata $(\mathrm{N}=2882)$ ont été utilisées pour décrire la relation poidslongueur. La longueur totale a varié de $5,8 \mathrm{~cm}$ à $18,4 \mathrm{~cm}$ et 4,9 à $10,8 \mathrm{~cm}$ respectivement pour $S$. m. melanotheron et E. fimbriata tandis que le poids total a varié de $3,40 \mathrm{~g}$ à $109,77 \mathrm{~g}$ et 1,70 à $46,24 \mathrm{~g}$ dans le même ordre. Les équations suivantes ont été obtenues : $\mathrm{Pt}=$ $0,056 \mathrm{Lt}^{2}, 543\left(\mathrm{r}^{2}=0,918\right.$, Ecart-type $=0,323, \mathrm{Pt}$ $=0,007 \mathrm{Lt}^{3,674}\left(\mathrm{r}^{2}=0,936\right.$, Ecart-type $=0,477$, respectivement pour $S$. m. melanotheron et $E$. fimbriata. Le coefficient d'allométrie b a été significativement différent de 3 pour les deux espèces (test $t$ de Student $: p<0,05$ ) et la croissance a été de type allométrie négative pour $S$. m. melanotheron ( $\mathrm{b}<3$, intervalle de confiance à $95 \%$ pour $\mathrm{b}: 2,220-2,866)$ et de type allométrie positive $(\mathrm{b}>3$, intervalle de confiance à $95 \%$ pour $\mathrm{b}: 3,197-4,151$ pour $E$. fimbriata).

\section{Taille à la première maturité}

La taille de première maturité sexuelle a été estimée à $12,20 \mathrm{~cm}$ et $10,80 \mathrm{~cm}$ respectivement pour les mâles et les femelles de $S$. m. melanotheron et 8,30 et $7,50 \mathrm{~cm}$ respectivement pour les mâles et les femelles de E. fimbriata (Figure 2). Il n'existe pas de différences significatives entre la taille de maturité des mâles et les femelles des deux espèces $(p>0,05)$.

\section{Paramètres de croissance}

La longueur asymptomatique $\mathrm{L} \infty$ et le coefficient de croissance $\mathrm{K}$ ont été évalués respectivement à $18,90 \mathrm{~cm}$ et $0,71 \mathrm{an}^{-1}$ pour $S$. m. melanotheron et $12,08 \mathrm{~cm}$ et $0,95 \mathrm{an}^{-1}$ pour E. fimbriata. L'indice de performance de croissance $\Phi$ ' a été de 2,40 pour $S . m$. melanotheron et 2,14 pour E. fimbriata. L'âge théorique $\mathrm{t}_{0}$ a été évalué à $-0,258$ pour $S . m$. melanotheron et - 0,216 pour E. fimbriata. La longévité potentielle $t_{\max }$ a été de 4,23 et 3,16 ans respectivement pour $S$. m. melanotheron et E. fimbriata. La Figure 3 indique les courbes de croissance superposées aux histogrammes de fréquence de taille. On note la présence de plusieurs courbes de croissance. Cette courbe de croissance indique par ailleurs que les poissons capturés appartenaient à 4 cohortes et 5 cohortes pour $S$. m. melanotheronet $E$. fimbriata, respectivement.

\section{Mortalité et taux d'Exploitation}

La mortalité totale a été estimée à 2,56 et $2,89 \mathrm{an}^{-1}$ pour $S . m$. melanotheron et $E$. fimbriata. La mortalité naturelle a été de 1,62 $\mathrm{an}^{-1}$ pour $S$. m. melanotheron et $2,22 \mathrm{an}^{-1}$ pour E. fimbriata (Figure 4). Les valeurs moyennes de $\mathrm{Z}$ et $\mathrm{M}$ ont donné une valeur de la mortalité par pêche $F$ de $0,94 \mathrm{an}^{-1}$ et $0,66 \mathrm{an}^{-1}$ respectivement pour $S$. m. melanotheron et $E$. fimbriata et le taux d'exploitation $\mathrm{E}$ a été de 0,37 et 0,23 pour $S$. m. melanotheron et $E$. fimbriata, respectivement.

\section{Recrutement}

Le recrutement est étendu sur toute l'année chez les deux espèces (Figure 5). Pour S. m. melalnotheron, le prémier et le plus grand pic est observé au début de la grande saison des pluies (mars-avril) alors que le deuxième pic est observé à la fin (juillet-août) de la même saison. Par contre, le premier pic de $E$. fimbriata a lieu en avril-juin pendant la grande saison des pluies et le deuxième et le plus grand pic en octobre-novembre pendant la décrue.

\section{Taille à la première capture / rendement relatif par recrue $Y^{\prime} / R$}

La longueur à la première capture $(\mathrm{Lc})$ a été estimée à $7,89 \mathrm{~cm}$ et $4,74 \mathrm{~cm}$ respectivement pour $S$. m. melanotheron et $E$. fimbriata (Figure 6). Le modèle de sélection logistique montre également que $25 \%$ des poissons $S$. m. melanotheron de $6,60 \mathrm{~cm}$ et $75 \%$ des poissons $S$. m. melanotheron de $9,9 \mathrm{~cm}$ sont 
actuellement pêchés. Ces chiffres sont respectivement de $4,01 \mathrm{~cm}$ et $5,48 \mathrm{~cm}$ pour $E$. fimbriata. La longueur optimale estimée a été de 10,74 et $6,79 \mathrm{~cm}$, respectivement pour $S . m$. melanotheron et $E$. fimbriata.

La courbe du rendement relatif par recrue $Y^{\prime} / \mathrm{R}$ en fonction du taux d'exploitation E (Figure 7) a donné un niveau optimal de taux d'exploitation Emax de 0,70 et 0,66 respectivement pour $S$. m. melanotheron et $E$. fimbriata. Les taux d'exploitation E0,1 (taux d'exploitation auquel l'augmentation marginale de $\mathrm{Y}^{\prime} / \mathrm{R}$ est $10 \%$ de son stock vierge) et E0,5 (taux d'exploitation sous lequel le stock est réduit de moitié de sa biomasse vierge) ont été également estimés. Les valeurs obtenues ont été respectivement de 0,55 et 0,34 pour $S$. $m$. melanotheron et 0,56 et 0,33 pour $E$. fimbriata.
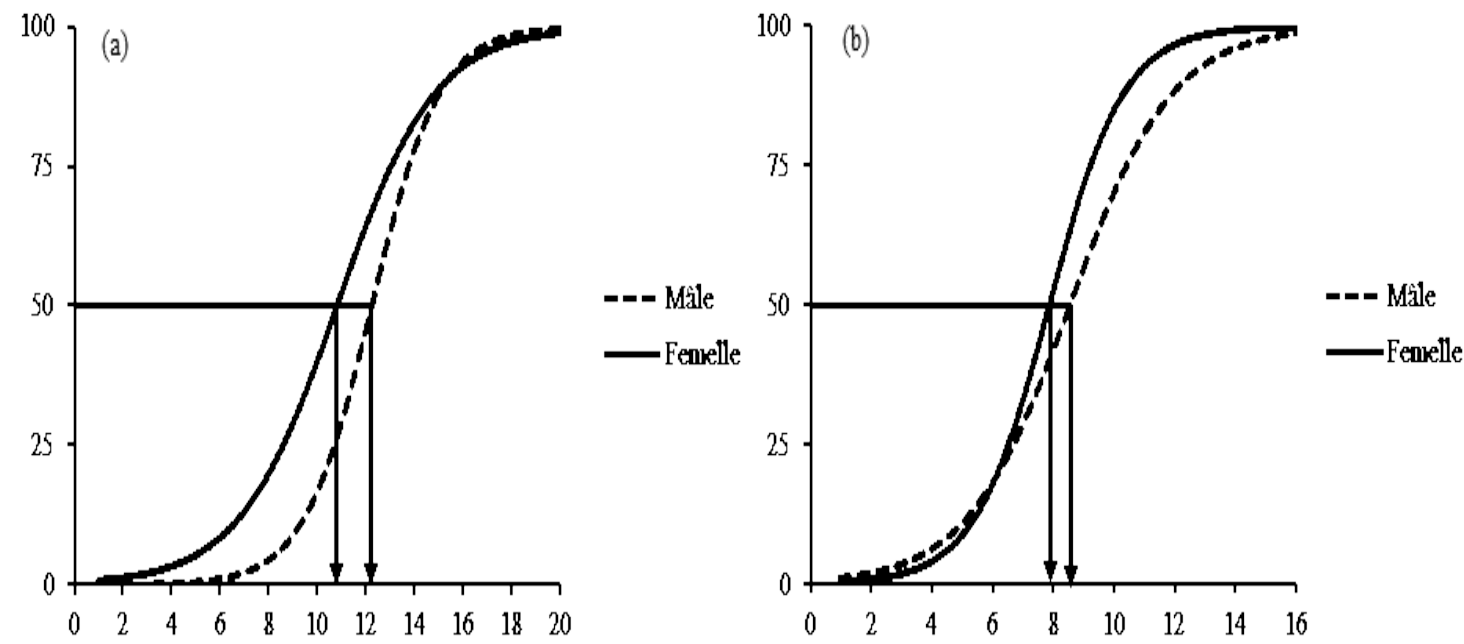

Figure 2: Taille de première maturité estimée de mâle et femelle de Sarotherodon melanotheron melanotheron (a) et Ethmalosa fimbriata (b) du lac Ahemé et ses chenaux

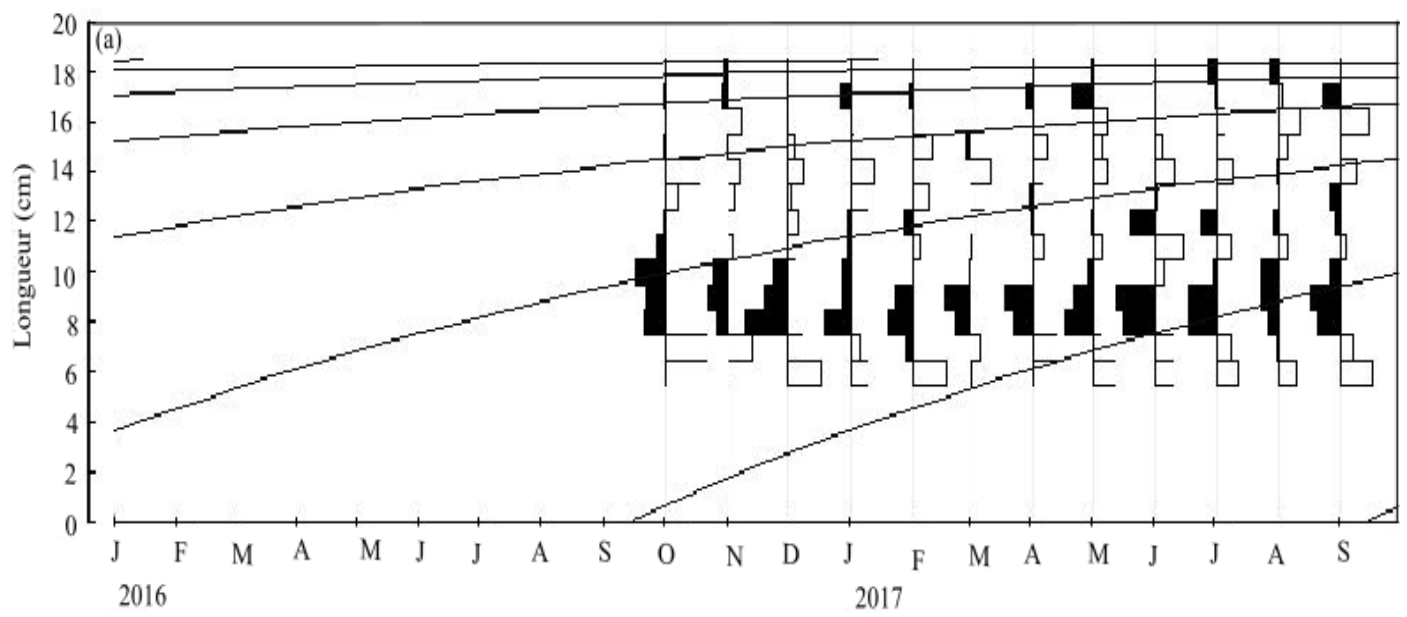




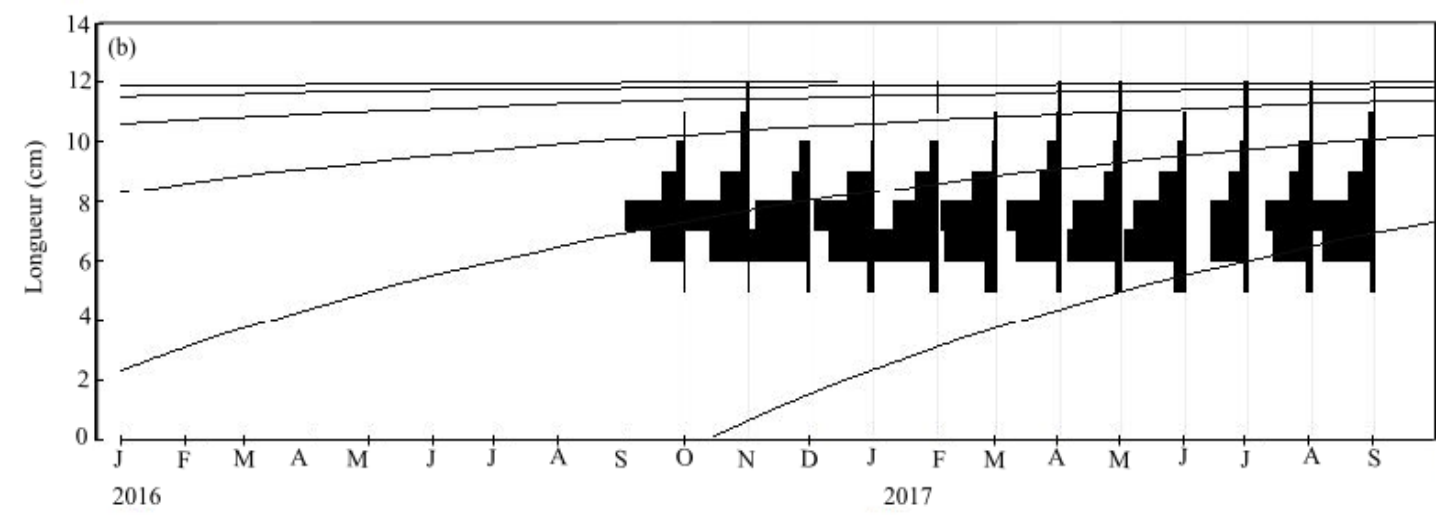

Figure 3 : Courbes de croissance obtenues à partir des histogrammes de fréquences de longueurs pour Sarotherodon melanotheron melanotheron (a) et Ethmalosa fimbriata (b) du lac Ahémé et ses chenaux.
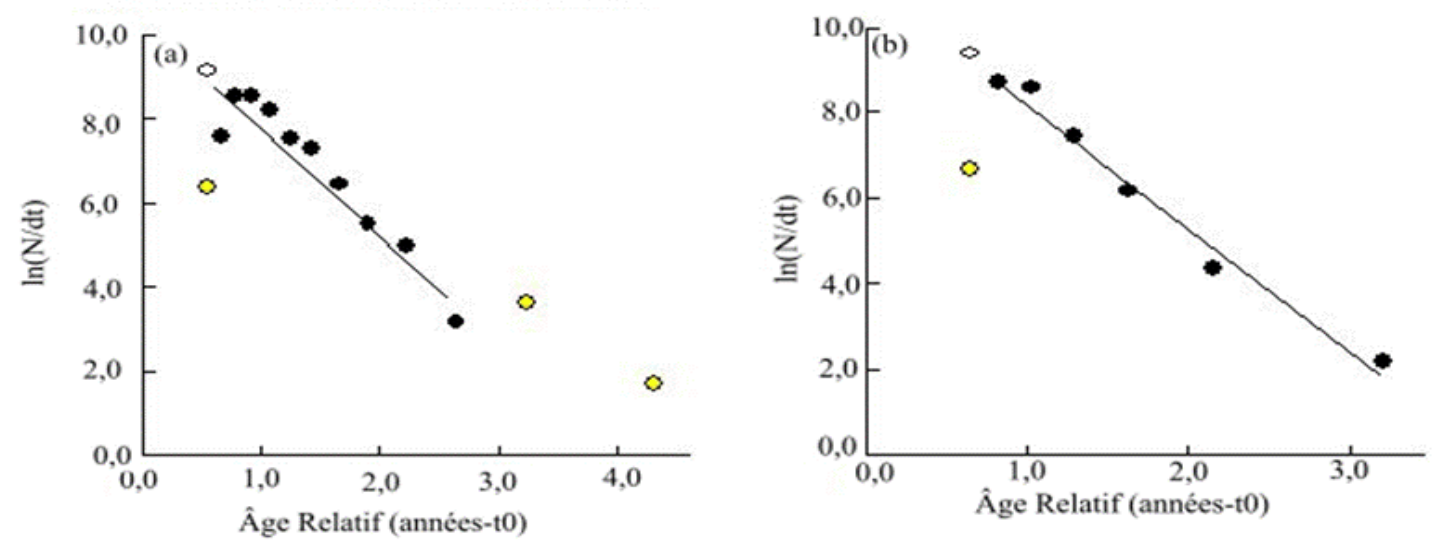

Figure 4: Les courbes de captures selon les longueurs pour (a) Sarotherodon melanotheron melanotheron et (b) Ethmalosa fimbriata du lac Ahémé et ses chenaux. Les points complets sont ceux utilisés pour calculer les paramètres de la ligne droite.
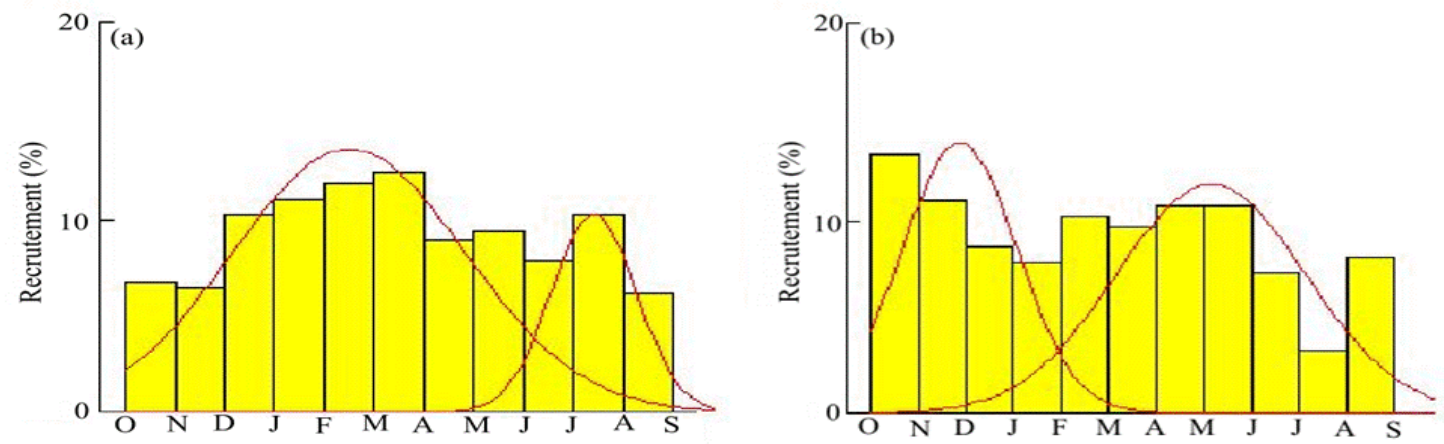

Figure 5 : Courbes de recrutement de Sarotherodon melanotheron melanotheron (a) et Ethmalosa fimbriata (b) du lac Ahémé et ses chenaux montrant deux pics par an. 

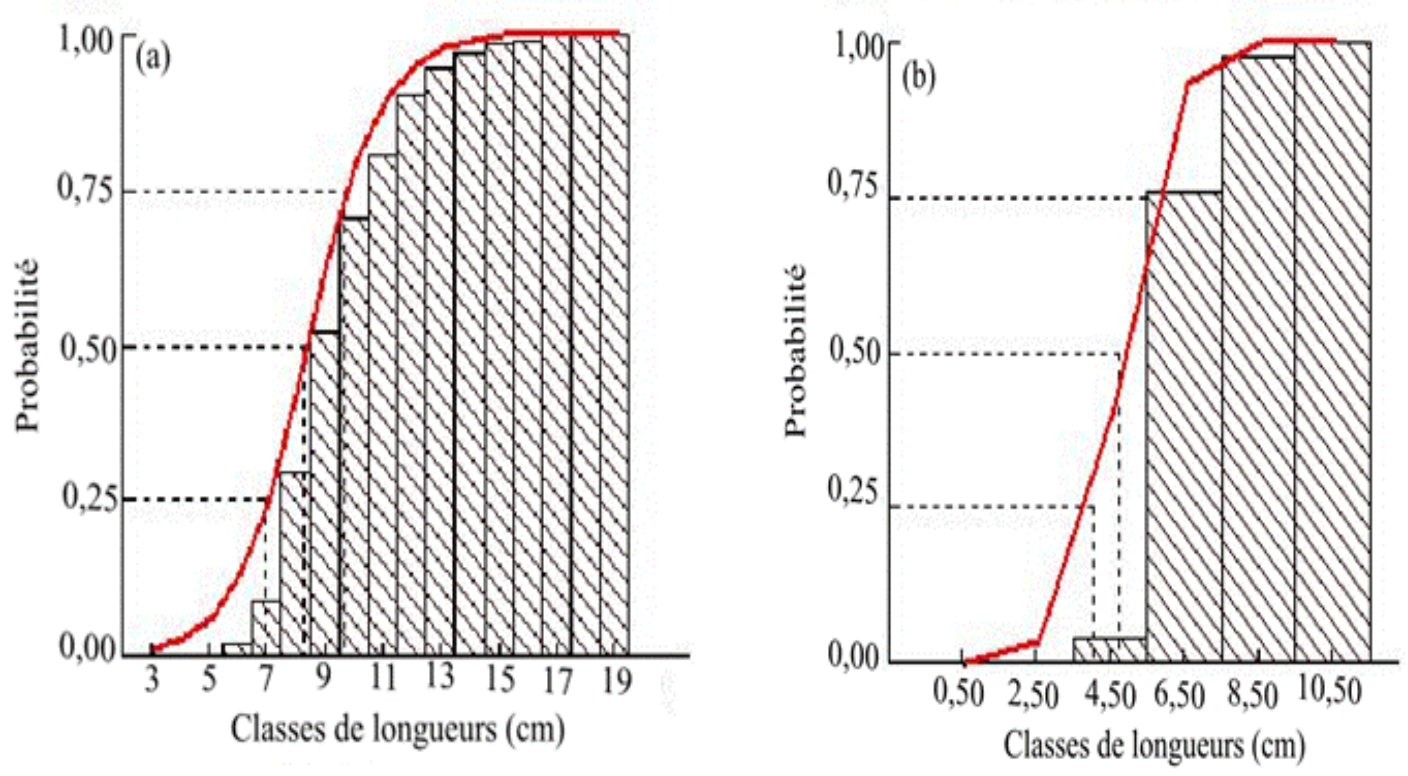

Figure 6: Probabilités de capture pour (a) Sarotherodon melanotheron melanotheron et (b) Ethmalosa fimbriata du lac Ahémé et ses chenaux estimées à partir de l'axe ascendant de la courbe de capture.
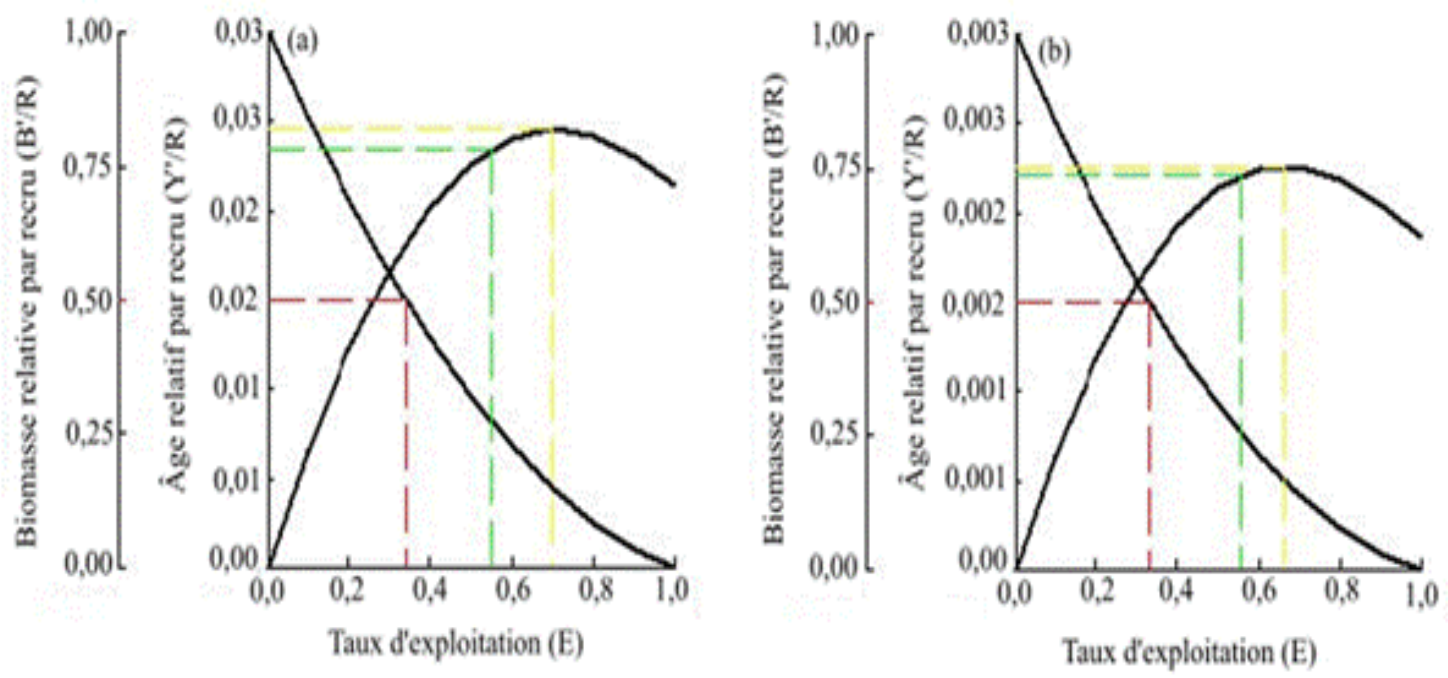

Figure 7 : Rendements relatifs par recruit et courbes de biomasses par recrût pour (a) Sarotherodon melanotheron melanotheron et (b) Ethmalosa fimbriata du lac Ahémé et ses chenaux en utilisant l'option Ogive. Les trois (03) lignes en pointillées correspondent respectivement à E0,5;E0,1 et Emax. 


\section{DISCUSSION}

Pour la présente étude, la réalisation des distributions de fréquence de taille de $S . m$. melanotheron et de E. fimbriataa montre que les échantillonnages ont pris en compte des spécimens jeunes et adultes, donc supposés représentatifs des populations. La relation poids-longueur est un outil important et très utilisé en biologie et physiologie et dans l'évaluation des stocks pour la gestion et la conservation des populations de poissons en milieux naturels (Lalèyè, 2006 ; Tah et al., 2012 ; Belhoucine, 2012 ; Hossainet al., 2012 ; Lederoun et al., 2016 ; Tenda et al., 2020). Elle permet d'estimer le poids d'un poisson connaissant sa longueur (ou inversement) lors de l'appréciation des rendements des pêches (Écoutin et Albaret, 2003 ; Froese, 2006 ; Froese et al., 2014 ; Tenda et al., 2020). L'analyse des relations poids-longueur a montré une croissance de type allométrie négative pour $S . m$. melanotheron et une croissance de type allométrie positive pour $E$. fimbriata dans le lac Ahémé et ses chenaux. Des travaux antécédents dans le lac Ahémé et d'autres écosystèmes du pays et de la sousrégion avaient déjà signalé ce type de croissance chez S. m. melanotheron. En effet, Niyonkuru et Lalayè (2012) avaient signalé une croissance allométrique négative pour $S$. $m$. melanotheron dans les lacs Ahémé et Nokoué. Au sud-ouest du Nigeria, dans le lac Eleiyele, Ayoade et Ikulala (2007) ont également signalé une croissance allométrique négative pour $S$. melanotheron. Contrairement aux auteurs précédents, Laléyé (2006) a signalé une croissance isométrique pour $S . \quad m$. melanotheron dans le fleuve Ouémé. Concernant E. fimbriata, les données sur le type d'allométrie dans le lac Ahémé et les autres écosystèmes du Bénin sont inexistantes. Néanmoins, Tenda et al. (2020) ont signalé une croissance allométrique positive pour $E$. fimbriata dans la lagune Mvassa de la basse Guinse, contrairement à Abowei (2009) qui a trouvé une croissance isométrique pour la même espèce dans la rivière Nkoro du Delta de Niger (Nigeria). D'autres auteurs ont également signalé une croissance isométrique pour E. fimbriata dans des eaux saumâtres (lagunes) et douces (lacs et fleuves) du Nigéria. Les variations dans relations poids-longueur ne sont pas inhabituelles chez les poissons des eaux tropicales (Niyonkuru et Lalayè, 2012 ; Lederoun et al., 2016 et Minoungou et al., 2020). Plusieurs facteurs peuvent être à la base d'une telle variation de la relation poidslongueur. En effet, les caractéristiques environnementales, les paramètres physicochimiques du milieu ainsi que la pression de pêche, le degré du parasitisme et toutes autres activités anthropiques sur le milieu sont autant de facteurs dont leurs variations peuvent influencer la croissance des espèces de poissons qui y vivent (Lederoun et al., 2016). Par exemple, une pression de pêche élevée peut réduire l'abondance des espèces, la concurrence intra spécifique pour la nourriture et pour l'espace, et par conséquent, améliorer l'état du poisson (masse plus grande pour une longueur de poisson donnée), tandis que des niveaux élevés de parasitisme peuvent affecter l'état des poissons (Lederoun et al., 2016).

La taille de première maturité sexuelle $\left(\mathrm{L}_{50}\right)$ est d'une grande importance pour déterminer le maillage optimal dans la gestion d'un stock de poissons (Lederoun et al., 2016). Dans la présente étude, les $\mathrm{L}_{50}$ obtenues pour les mâles $(12,20 \mathrm{~cm}$ et $10,80 \mathrm{~cm})$ et les femelles $(10,30 \mathrm{~cm}$ et $7,50 \mathrm{~cm})$ respectivement pour $S$. $m$. melanotheron et $E$. fimbriata, étaient relativement petites pour des espèces pouvant atteindre respectivement $250 \mathrm{~mm}$ et $350 \mathrm{~mm}$ de longueur standard (SL) (Teugels et Thys van den Audenaerde, 2003 ; Gourène et Teugels, 2003). Les espèces de poissons des systèmes fluviaux-lagunaires tropicaux sont reconnues pour leur maturité très rapide, qui est considérée comme une réponse adaptative aux conditions instables de ces systèmes (Lederounet al., 2015). La maturité sexuelle relativement précoce constatée chez ces deux espèces peut être attribuée en majeure partie à la pression de la pêche et à la destruction de l'habitat par les diverses techniques de pêches (acadjas et des barrages à nasses) développées sur le lac Ahémé et ses chenaux. Pour chaque espèce, la taille de première maturité sexuelle enregistrée est supérieure à la taille de première capture $(7,89 \mathrm{~cm}$ et $4,74 \mathrm{~cm}$ respectivement 
pour S. m. melanotheron et E. fimbriata). Au lac Ahémé et ses chenaux, les populations de $S$. $m$. melanotheron et $E$. fimbriata sont menacées, les poissons sont pêchés sans avoir tous eu la chance de se reproduire une fois.

L'évaluation et la gestion des stocks reposent généralement sur l'estimation des paramètres de croissance et de mortalité des populations (Alhassan et Armah, 2011; Lederoun et al., 2015). Pour cette étude, les longueurs asymptomatiques $(\mathrm{L} \infty)$ ont été de $18,90 \mathrm{~cm}$ et $12,08 \mathrm{~cm}$ respectivement pour $S$. $m$. melanotheron et $E$. fimbriata. Pour chaque espèce, la $L \infty$ est raisonnable lorsqu'elle est proche de la longueur maximale de poissons observée dans les échantillons et que le taux est inférieur à zéro. Quant aux valeurs de K, elles doivent être comprises entre zéro (0) et un (01) pour les poissons à longue durée de vie et un excès de un (01) pour les espèces à durée de vie courte. Les valeurs des paramètres de croissances estimés respectent les critères suscités pour les deux espèces. Pour $S . m$. melanotheron, la valeur de $\mathrm{L} \infty(18,90 \mathrm{~cm})$ obtenue pour cette étude est largement inférieure à celles obtenues par Niyonkuru (2007) dans ce même écosystème $(22,5 \mathrm{~cm})$ et au lac Nokoué $(24,1$ à $26,7 \mathrm{~cm}$ ) ainsi qu'à celle de Lederoun et al. (2015) au lac Toho (Bénin) $(21,53 \mathrm{~cm})$. Une comparaison de la L $\infty$ de $S . m$. melanotheron avec celles trouvées dans la sous-région par d'autres auteurs pour la même espèce a permis de constater que cette valeur est inférieure à toutes les valeurs trouvées jusque-là (22 à $37 \mathrm{~cm}$ ) Villanueva, 2004 ; Niyonkuru et al., 2007). Néanmoins, la vitesse de croissance $\mathrm{K}$ obtenue pour $S . \quad m$. melanotheron $\left(0,71 \mathrm{an}^{-1}\right)$ est meilleure par rapport celles trouvées par d'autres auteurs $\left(0,16\right.$ à $\left.0,6 \mathrm{an}^{-1}\right)$ (Fagade, $1974 ;$ Villanueva, 2004 ; Niyonkuru, 2007 et Lederoun et al., 2015). Contrairement aux auteurs précédents, Niyonkuru et al. (2007) et Niyonkuru (2007) ont trouvé des $\mathrm{K}$ plus élevés $\left(0,74\right.$ à $\left.1,78 \mathrm{an}^{-1}\right)$ respectivement au Togo dans le lac de Lomé, Sakumo (Ghana), au lac Nokoué et dans les acadjas du lac Nokoué (Bénin). Quant à $E$. fimbriata, la longueur asymptomatique $\mathrm{L} \infty$ obtenue $(12,08 \mathrm{~cm})$ est faible comparativement à celle trouvée par Niyonkuru et al. (2007) au lac Noukoué $(\mathrm{L} \infty=22 \mathrm{~cm})$. D'autres auteurs ont obtenu des $L \infty$ très élevés dans la sousrégion $(24,5$ à $40,8 \mathrm{~cm})$ comparativement à celle trouvée dans la présente étude (AmaAbasi et al., 2004 ; Panfili et al., 2004, Faye et al. 2014). Cependant, le K obtenu $\left(0,95 \mathrm{an}^{-1}\right)$ est meilleur par rapport à ceux trouvés par tous les auteurs sus-cités pour E. fimbriata $(\mathrm{K}=0,25$ - $0,90 \mathrm{an}^{-1}$ ) à l'exception Niyonkuru et al. (2007) qui ont obtenu une valeur de K plus élevés (respectivement $0,96 \mathrm{an}^{-1}$ et $1 \mathrm{an}^{-1}$ en Côte d'Ivoire et au lac Nonkoué). Les variations des paramètres de croissances d'un écosystème à un autre peuvent être expliquées par les conditions environnementales locales, des différences génétiques, des différences des paramètres physiques et chimiques, de la disponibilité de nourriture et des maladies. La réduction de la longueur asymptomatique $\mathrm{L} \infty$ des deux espèces pourrait s'expliquer par l'activité de pêche intensive. Au lac Ahémé, divers engins et techniques de pêches sont utilisés pour exploiter les ressources halieutiques. Il en existe de différentes gammes pour capturer les diverses tailles de poissons (Gnidete et al., 2017). C'est ce qui expliquerait l'absence des spécimens de grandes tailles pour les deux espèces dans le lac Ahémé et ses chenaux. Cette hypothèse est confirmée par le travail de Niyonkuru (2007) qui a trouvé que la population de $S$. m. melanotheron du lac Nokoué présentait des paramètres de croissances plus élevés que ceux de la population du lac Ahémé. Selon cet auteur, les meilleures valeurs des paramètres de croissance observées au lac Nokoué peuvent être attribuées à la présence de nombreux acadjas dans ledit lac. En effet, les acadjas constituent des frayères, des zones de protection et une source de nourriture pour certaines espèces de poisson. Ils permettent ainsi de limiter l'influence des activités de pêches sur les populations de poissons et par conséquent, les spécimens de grande taille ne sont pas tous éliminés. La réduction de la longueur asymptotique de $S$. m. melanotheron dans le lac Ahémé $(18,90 \mathrm{~cm}$ pour la présente étude contre $22,5 \mathrm{~cm}$ trouvé par Niyonkuru en 2007) peut donc être expliquée par l'augmentation des pressions de pêche due à 
une augmentation continue du nombre de pêcheurs.

L'indice de performance de croissance $\Phi^{\prime}$ est le meilleur outil de comparaison des paramètres de croissances d'une espèce (Nomwine et al., 2018). Les indices de performance de croissance $\Phi^{\prime}$ calculés dans la présente étude, 2,40 pour $S$. m. melanotheron et 2,14 pour E. fimbriata, se situent dans la fourchette de ceux signalés dans la littérature. En effet, des résultats comparables ont été obtenus par d'autres auteurs pour $S$. $m$. melanotheron. Par exemple Dadebo et al., (1983) ont obtenu respectivement 2,24 dans la lagune de Lagos (Nigeria) et 2,73 dans la lagune d'Ebrié (Côte d'Ivoire), tandis que Niyonkuru (2007) a enregistré 2,75 et 2,42 respectivement dans le lac Nokoué et le lac Ahémé (Bénin) ainsi que Lederoun et al. (2015) qui ont enregistré 2,43 au lac Toho (Bénin) pour S. m. melanotheron. Concernant E. fimbriata, Niyonkuru et al. (2007) ont trouvé au lac Nokoué (Bénin) un $\Phi^{\prime}(2,67)$ supérieur à celui de la présente étude, de même que Faye et al. (2014), qui ont enregistré respectivement 2,67 et 2,48 en mer et estuaire au Sénégal. Néanmoins, plusieurs auteurs ont trouvé pour la même espèce des $\Phi$ ' comparables à celui obtenu dans cette étude. En effet, Ama-Abasi et al. (2004), et Panfili et al. (2004) ont enregistré respectivement 2,76 (Côte d'Ivoire) ; 2,47 ; 2,49 et 2,94 (Nigeria) ; 2,62 (Sierra Leone) et 2,54 (Sénégal). D’après Baijot et Moreau (1997 in Lederoun 2016), les valeurs moyennes de $\Phi^{\prime}$ pour la majorité des poissons importants en Afrique variaient entre 2,65 et 3,32 . Selon ces auteurs, les $\Phi^{\prime}$ appartenant à cette intervalle $(2,65$ et 3,32$)$ sont considérés comme de faibles taux de croissance. Dans cette étude, les taux de croissance des deux espèces $\Phi^{\prime}$ sont situés dans cette fourchette ; ainsi, les poissons du lac Ahémé et ses chenaux pourraient également être considérés comme présentant une croissance lente.

Les mortalités totale $\left(Z=2,89 \mathrm{an}^{-1}\right)$ et naturelle estimée $\left(\mathrm{M}=2,22 \mathrm{an}^{-1}\right)$ de $E$. fimbriata sont plus élevées que celles de $S$. $m$. melanotheron $\left(\mathrm{Z}=2,56 \mathrm{an}^{-1}\right.$ et $\left.\mathrm{M}=1,62 \mathrm{an}^{-1}\right)$, contrairement à la mortalité par pêche qui est plus élevée chez $S$. m. melanotheron $(\mathrm{F}=0,94$ $\left.\mathrm{an}^{-1}\right)$ que chez $E$. fimbriata $\left(\mathrm{F}=0,66 \mathrm{an}^{-1}\right)$. Au lac Ahémé et ses chenaux, la population de $S$. $m$. melanotheron serait plus sollicitée plus que celle de E. fimbriata. Niyonkuru et al. (2007) ont obtenu d'autres résultats $\left(\mathrm{Z}=2,3 \mathrm{an}^{-1}\right.$ et $\mathrm{M}$ $=1,5 \mathrm{an}^{-1}$ pour $S$. melanotheron $; \mathrm{Z}=2,3 \mathrm{an}^{-1}$ et $\mathrm{M}=2 \mathrm{an}^{-1}$ pour $E$. fimbriata) au lac Nokoué (Bénin), de même que Lederoun et al. (2015) $\left(\mathrm{Z}=1,95 \mathrm{an}^{-1}\right.$ et $\mathrm{M}=1,37 \mathrm{an}^{-1}$ pour $S$. melanotheron) au lac Toho. Niyonkuru (2007) a également signalé des mortalités plus ou moins élevées $\left(Z=1,66-2,41 \mathrm{an}^{-1}\right.$ et $\mathrm{M}=1,28$ $-1,58 \mathrm{an}^{-1} ; \mathrm{Z}=2,1 \mathrm{an}^{-1}$ et $\mathrm{M}=1,26 \mathrm{an}^{-1}$ ) chez $S$. melanotheron respectivement aux lacs Nokoué et Ahémé. Selon cet auteur, ces mortalités seraient liées en grande partie à la technique de pêche appelée localement « djétowlè) qui consiste à fouiller la vase pour dénicher les poissons après avoir lancé le filet épervier. Les poissons ayant échappé à la capture mourraient par colmatage des branchies. Pour les deux espèces, les mortalités totales et celles naturelles estimées dans cette étude sont supérieures aux valeurs existantes sur le lac Ahémé et d'autres écosystème du Bénin (Niyonkuru, 2007 ; Niyonkuru et al., 2007 ; Lederoun et al., 2015). Cela pourrait être attribué aux perturbations écologiques du lac Ahémé dues aux encombrements et comblement dudit lac par les acadjas. Cette hypothèse a été confirmée par Niyonkuru (2007) qui avait constaté que les mortalités de la population de $S$. melanotheron du lac Nokoué hors acadjas $\left(\mathrm{Z}=1,66 \mathrm{an}^{-1}\right.$ et $\mathrm{M}=1,28$ $\mathrm{an}^{-1}$ ) sont plus faibles que celles au sein des acadjas $\left(Z=2,41 \mathrm{an}^{-1}\right.$ et $\left.\mathrm{M}=1,58 \mathrm{an}^{-1}\right)$.

Le rapport $\mathrm{M} / \mathrm{K}$ est utilisé pour déterminer la fiabilité de la mortalité naturelle M (Beverton et Holt, 1959 in Lederoun, 2016). Selon ces auteurs, ce rapport est compris entre 1,12 et 2,50 pour la plupart des poissons. Les rapports $\mathrm{M} / \mathrm{K}$ obtenus pour cette étude, 2,28 et 2,34 respectivement pour $S$. m. melanotheron et $E$. fimbriata, se situaient dans cette fourchette et suggéraient que les mortalités naturelles estimées pour ces espèces étaient fiables. Des valeurs comparables du rapport $\mathrm{M} / \mathrm{K}$ sont obtenues par d'autres auteurs. En effet, Niyonkuru et al. (2007) ont trouvé 1,87 
et 2 respectivement pour $S . m$. melanotheron et E. fimbriata au lac Nokoué. De même, Niyonkuru (2007) a obtenu pour $S . \quad m$. melanotheron 2 à 2,33 au lac Nokoué et 2,42 au lac Ahémé.Lederoun et al. (2015) ont également obtenu 2,36 pour $S . \mathrm{m}$. melanotheron au lac Toho. La mortalité naturelle reste supérieure à la mortalité par pêche pour S. m. melanotheron et E. fimbriata dans le lac Ahémé et ses chenaux. Les mortalités naturelles élevées peuvent être imputées à la dégradation des habitats, aux modifications des paramètres physicochimiques et écologiques du lac et à la prédation.

Une comparaison de la mortalité naturelle $(\mathrm{M})$ par rapport à celle par pêche $(\mathrm{F})$ permet d'évaluer l'état ou le niveau d'exploitation d'une espèce. Pour Pauly (1987 in Niyonkuru, 2007), l'activité de pêche est conservatrice lorsque $\mathrm{F}=0,4 \mathrm{M}$; Gulland (1971 in Niyonkuru, 2007) propose par contre que l'exploitation est optimale lorsqu'il y a égalité entre $\mathrm{M}$ et $\mathrm{F}$. Les mortalités par pêche $\mathrm{F}$ $\left(0,94 \mathrm{an}^{-1}\right.$ et $0,66 \mathrm{an}^{-1}$ respectivement pour $S$. $m$. melanotheron et E. fimbriata) sont inférieures aux mortalités naturelles $\mathrm{M}\left(1,62 \mathrm{an}^{-1}\right.$ pour $S$. $m$. melanotheron et $2,22 \mathrm{an}^{-1}$ pour $E$. fimbriata). Les produits $0,4 \times \mathrm{M}$ donnent respectivement $0,65\left(<\mathrm{F}=0,94 \mathrm{an}^{-1}\right)$ et $0,89(>$ $\mathrm{F}=0,66 \mathrm{an}^{-1}$ ) pour $S . m$. melanotheron et $E$. fimbriata. Ainsi, le stock de $S . \quad m$. melanotheron dans le lac Ahémé et ses chenaux peut être supposé surexploité alors que celui de E. fimbriata reste non surexploité.

La courbe de rendement relatif par recrue $Y^{\prime} / \mathrm{R}$ est plus utilisée pour décider de la surexploitation ou non des stocks de poissons dans un écosystème aquatique. En effet, dans la présente étude, les taux d'exploitation actuels des deux espèces $(0,37$ pour $S . \quad m$. melanotheron et 0,23 pour $E$. fimbriata) sont inférieurs aux taux d'exploitation prévus pour donner le maximum de $\mathrm{Y}^{\prime} / \mathrm{R}(0,70$ pour $S$. $m$. melanotheron et 0,66 pour E. fimbriata). Une comparaison des taux d'exploitation actuels avec les estimations des taux qui maintiennent $50 \%$ de la biomasse du stock, E 0,5 (0,34 pour $S$. m. melanotheron et 0,33 pour E. fimbriata), a permis de constater que le taux d'exploitation actuel de $S$. m. melanotheron est supérieur à son $\mathrm{E} 0,5$ alors que le taux d'exploitation actuel de $E$. fimbriata reste inférieur à son E 0,5. Ces résultats confirment que la population de $S . m$. melanotheron du lac Ahémé est soumise à une surexploitation, contrairement à E. fimbriata dont le stock n'est pas encore surexploité, cela corrobore une fois de plus la déduction faite lors de l'examen du produit $0,4 \times \mathrm{M}$ déterminé plus haut. Ces résultats sont conformes à ceux trouvés par Niyonkuru (2007) qui stipulent qu'au lac Ahémé, les populations de poissons en général et les Cichlidae en particulier $(S$. melanotheron et $H$. fasciatus et $C$. guineensis) sont surexploitées. Les modèles utilisés ont montré que la population de E. fimbriata du lac Ahémé et ses chenaux reste non surexploitée. Contrairement aux précédents auteurs, Barry et Tegner (1989 in Niyonkuru, 2007) ont proposé le rapport $\mathrm{Z}$ / K comme modèle/outil d'appréciation du niveau d'exploitation. Ce modèle compare la mortalité totale $\mathrm{Z}$ à la vitesse de croissance $K$ au sein d'une population. Il est souhaitable qu'il y ait un équilibre entre la mortalité et la croissance $(\mathrm{Z} / \mathrm{K}=1)$. Lorsque $\mathrm{Z} / \mathrm{K}=2$, la population est supposée légèrement exploitée, mais lorsque $\mathrm{Z} / \mathrm{K}>2$, il y a prédominance de la mortalité sur la croissance, et dans ce cas, la population semble être soumise à une surexploitation. Les rapports $\mathrm{Z} / \mathrm{K}$ obtenus $(3,60$ et 3,04 respectivement pour $S$. m. melanotheron et $E$. fimbriata) sont tous supérieurs à 2 , la mortalité totale prédomine sur la croissance pour les deux espèces. Les sujets meurent (que ça soit par pêche ou non) plus qu'ils grandissent, il y a donc diminution du stock; le renouvellement du stock (biomasse) est inférieur à la perte par mortalité. Ce modèle a permis de confirmer une fois de plus la surexploitation de la population de $S$. m. melanotheron du lac Ahémé et ses chenaux et stipule qu'il peut y avoir surexploitation de la population de $E$. fimbriata. Même si l'effort de pêche sur la population E. fimbriata $(\mathrm{E}=0,23<\mathrm{E} 0,50=$ 0,33 ) est dans les normes d'une exploitation rationnelle, l'espèce est menacée. Si des mesures de protection par assainissement de l'écosystème, l'enlèvement systématique des engins et techniques de pêche dévastateurs, la 
sensibilisation et la répression sur les méthodes de pêche durables ne sont pas entreprises, ce résultat (non surexploitation apparente de $E$. fimbriata) peut changer facilement à une surexploitation dans le futur. Pour une gestion rationnelle du lac, il est très indispensable que le maillage des filets soit revu avec des mesures de répression (Niyonkuru, 2007), que des zones de réserves biologiques d'interdiction de pêches soient créés pour constituer des frayères afin de permettre un renouvellement permanent des stocks de poissons du lac Ahémé et ses chenaux. De même, il est nécessaire d'envisager la diversification des activités des pêcheurs et de réhabiliter les plans d'eau à travers les opérations visant à augmenter la profondeur et à réduire leur comblement sous toutes les formes.

Le recrutement est fortement influencé par les facteurs environnementaux (Welcomme, 2001). Des études sur différents bassins versants ont montré que la saisonnalité de la reproduction des espèces de poissons est influencée par la température de l'eau, les précipitations et les conditions hydrologiques (Dadebo et al., 2003 ; Lederoun et al., 2015). Le succès de la reproduction dépend d'une période favorable à la production de jeunes individus qui détermineront la pérennité de l'espèce (Lowe-McConnell, 1999 in Niyonkuru, 2007). Dans cette étude, le recrutement a été continu sur toute l'année pour les deux espèces. Pour S. m. melalnotheron, le plus grand pic est observé entre mars et avril alors que le deuxième pic est observé entre juillet et août. Pour E. fimbriata, le premier pic est observé entre avril et juin et le deuxième et le plus grand pic entre octobre et novembre. Pendant les saisons des pluies, les berges sont inondées entrainant l'expansion de l'habitat dans les zones inondées. A cela s'ajoute la disponibilité accrue des ressources, les juvéniles de $S$. m. melanotheron et de $E$. fimbriata trouveraient certainement un abri et pourraient assurer leur propre alimentation. Contrairement aux résultats de cette étude, Niyonkuru (2007) avait trouvé qu'au lac Ahémé, le grand pic de recrutement de $S$. $m$. melanotheron est observé entre septembre et octobre (saison de crue) et le petit pic entre mai et juin. Le changement de périodes de reproduction chez $S$. m. melanotheron serait dû en grande partie aux changements climatiques. Les pluies tardent à tomber et tombent pendant des périodes relativement courtes. Les Cichlidae étant connus de leur adaptabilité aux variations des conditions du milieu, la population de $S$. $m$. melanotheron du lac Ahémé se serait certainement adaptée aux changements du climat et aux pressions anthropiques. Le grand pic de recrutement de E. fimbriata observé pendant la décrue (Octobre-Novembre) se justifierait par l'augmentation de la salinité du lac lac Ahémé et ses chevaux. En effet, pendant la décrue, l'arrivée de l'eau de mer dans le lac Ahémé et ses chenaux entraine une augmentation de la salinité de l'eau, le milieu devient propice pour la survie et la croissance de E. fimbriata.

\section{Conclusion}

Le stock de $S$. m. melanotheron dans le lac Ahémé et ses chenaux est surexploité. Quant à E. fimbriata, son stock reste encore non surexploité ; néanmoins le renouvellement dudit stock (biomasse) est inférieur à la perte par mortalité, donc l'état d'exploitation rationnelle de E. fimbriata n'est qu'apparente et peut changer rapidement en surexploitation. Il convient de prendre des mesures de protection par assainissement de l'écosystème à travers l'enlèvement systématique des engins et techniques de pêche dévastateurs, la répression, la sensibilisation des populations sur les méthodes de pêches durables et de créer des zones de réserves biologiques d'interdiction de pêches pour constituer des frayères afin de permettre un renouvellement permanent des stocks de poissons.

\section{CONFLIT D'INTÉRÊTS}

Les auteurs déclarent qu'ils n'ont pas de conflit d'intérêts.

\section{CONTRIBUTIONS DES AUTEURS}

CCV est le responsable de l'étude; il a contribué à la collecte des données et à la rédaction de l'article; DL et TAH ont contrôlé les résultats des analyses; ISB a participé à la collecte des données sur le terrain. 
SAM et DA ont fait la relecture de l'article ; NMG a contribué au financement de la collecte des données sur le terrain; PL est le superviseur général des activités dans le cadre de ce manuscrit.

\section{REMERCIEMENTS}

$\checkmark$ Laboratoire d'Hydrobiologie et d'Aquaculture, Faculté des Sciences Agronomiques, Université d'Abomey-Calavi,

$\checkmark$ Laboratoire de Recherche en Aquaculture et en Biologie et Ecologie Aquatiques, Ecole d'Aquaculture de Vallée, Université Nationale d'Agriculture (UNA), Bénin.

Agence pour le Développement intégré de la zone Economique du Lac Ahémé et ses Chenaux (ADELAC).

\section{RÉFÉRENCES}

Abowei JFN. 2009. The Morphology, Abundance, Condition Factor and Length-weight Relationship of Ethmalosa fimbriata (Bowdish 1825) from Nkoro River Niger Delta, Nigeria .Advance. Journal of Food Science and Technology, 1(1): 51 - 56. DOI: https://www.researchgate.net/publication $/ 266260549$

Adandedjan D. 2012. Diversité et déterminisme des peuplements de macro invertébrés benthiques de deux lagunes du sud-Bénin : la lagune de Porto-Novo et la lagune côtière Doctorat unique en Sciences Agronomiques de 1'Université d'Abomey-Calavi (2012), 239 p.

Alhassan EH, Armah AK. 2011. Population dynamics of the African river prawn, Macrobrachium vollenhovenii, in Dawhenya impoundment. Turkish Journal of Fisheries and Aquatic Sciences, 11(1): 113 - 119. DOI: https://dergipark.org.tr/en/download/artic le-file/141738

Ama-Abasi D, Holzloehner S, Enin U, 2004. The dynamics of the exploited population of Ethmalosafimbriata (Bowdich, 1825, Clupeidae) in the Cross River Estuary and adjacent Gulf of Guinea. Fisheries Research, 68 (1-3) : 225 - 235. DOI : http://dx.doi.org/10.1006/gcen.1998.710 5

Amoussou E. 2014. Lacs et lagunes anciens du Sud-Ouest Bénin, état des lieux. IUCN Bénin, Rapport d'étude : 122-201.

Ayoade AA, Ikulala AOO. 2007. Length weight relationship, condition factor and stomach contents of Hemichromis bimaculatus, Sarotherodon melanotheron and Chromido tilapia guentheri (Perciformes: Cichlidae) in Eleiyele Lake, Southwestern Nigeria. Revista de Biologia Tropical, 55 (3-4) : 969 - 977. DOI: http://dx.doi.org/10.15517/rbt.v55i34.5970

Belhoucine F, 2012. Étude de la biologie de la croissance et de la reproduction d'un poisson téléostéen le merlu (Merluccius merluccius L., 1758) et son utilisation comme indicateur biologique de la pollution par les métaux lourds (Zinc, Plomb et Cadmium) dans la baie d'Oran (Algérie). Diss. Thèse de doctorat: Science de l'environnement. Oran: Université d'Oran, $325 \mathrm{p}$.

Dadebo E, Ahlgren G, Ahlgren I. 2003. Aspects of reproductive biology of Labeo horie Heckel (Pisces: Cyprinidae) in Lake Chamo. Ethiopia. African Journal of Ecology, 41: 31-38. DOI : https://doi.org/10.1046/j.1365-

2028.2003.00404.x

Écoutin JM, Albaret JJ, 2003. Relation longueur-poids pour 52 espèces de poissons des estuaires et lagunes de l'Afrique de l'Ouest. Cybium, 27 (1) : 3 9.

https://www.researchgate.net/publication /294493366_Length-

weight_relationship_of_52_fish_species _from_West_African_estuaries_and_lag oons

Faye A. Sarr A. Thiaw M. Ba K. Ndiaye I. Lazar N. Thiaw OT. 2014. Contribution to the Study of the Growth of the Bonga Ethmalosa fimbriata (Bowdich) in Senegalese Coastal Waters. Journal of Biology and Life Sciences, 5 (1): 82. 
https://scholar.google.com/citations?user $=4 \mathrm{fEhLy} 8 \mathrm{AAAAJ} \& \mathrm{hl}=\mathrm{fr}$

Froese R. Thorson JT. Reyes RB. 2014. A bayesi an approach for estimating lengthweight Relationship in fishes. Journal of Applied Ichthyology, 30: 78 - 85. https://onlinelibrary.wiley.com/doi/abs/1 0.1111/jai.12299

Froese R. 2006. Cube law, condition factor and weight-length relationships: History, meta-analysis and recommendations. Journal of Applied Ichthyology, 22: 241 253.

https://onlinelibrary.wiley.com/doi/10.11 11/j.1439-0426.2006.00805.x

Gnidete F. Houssou CS. Gibigaye M. Vissin EW. Cledjo P. Laleye P. 2017. Systèmes d'exploitation halieutiques dévastateurs des ressources naturelles dans le complexe fluvio-lacustre Couffo-AhéméAhô-lagune côtière, Sud-Ouest du Bénin. Rev. Ivoir. Sci. Technol., 30: 160 - 181.

Lederoun D. Chikou A. Vreven E. Snoeks J. Moreau J. Vandewalle P. Lalèyè P. 2015. Population parameters and exploitation rate of Sarotherodon melanotheron melanotheron Rüppell 1852 (Cichlidae) in Lake Toho, Benin. Journal of Biodiversity and Environmental Sciences, 6: $\quad 259 \quad 271$. https://orbi.uliege.be/bitstream/2268/249 761/2/African\%20J.\%20Aquatic\%20Sci ence $\% 20 \% 282016 \% 29$ Vandewalle.pdf

Lederoun D. Laleye P. Vreven E. Vandewalle P. 2016. Length-weight and length weight relationships and condition factor for 30 actinopterygian fish from the mono basin (Benin and Togo, West Africa). Cybium 40(4): 267-274. DOI: https://doi.org/10.2989/16085914.2016.1 169988

Lederoun D. Vandewalle P. Brahim AA. Moreau J. Lalèyè PA. 2016. Population parameters and exploitation rate of Sarotherodon galilaeus galilaeus (Cichlidae) in Lakes Doukon and Togbadji, Benin. African Journal of Aquatic Science, 41 (2) : 151 - 160. DOI: https://doi.org/10.2989/16085914.2016.1 169988
LOwerre-barbieri SK. Brown-Peterson NJ. Murua H. 2011. Emerging issues and methodological advances in fisheries reproductive biology. Marine and Coastal Fisheries, 3(1) : 32 - 51. DOI: 10.1080/19425120.2011.555725

Minoungou M, Ouedraogo R, Nomwine DA, Oueda A. 2020. Relation longueur-poids et facteur de condition de sept espèces de poisson du réservoir de Samandeni avant son ouverture à la pêche (Burkina Faso). Journal of Applied Biosciences, 151: $\quad$ 15559-15572. DOI: https://doi.org/10.35759/JABs.151.5

Nomwine DA. Raymond O. Adama O. 2018. Relation poids-longueur et facteur de condition de Clarias anguillaris et Sarotherodon galilaeus pêchées dans le lac Bam et le réservoir de la Kompienga au Burkina Faso Int. J. Biol. Chem. Sci., 12(4) : 1601-1610. DOI: https://dx.doi.org/10.4314/ijbcs.v12i 4.8

Niyonkuru C, Lalèyè PA, Villanueva MC, Moreau J. 2007. Population parameters of main fish species of lake Nokoué, Benin. Journal of Afro Tropical Zoology, 3 (Special issue): 149-155. https://www.researchgate.net/publication 1255711836

Niyonkuru C. 2007. Etude comparée de l'exploitation et de la démographie des poissons Cichlidés dans les lacs Nokoué et Ahémé au Bénin. Thèse de Doctorat en Gestion de l'Environnement, Université d'Abomey-Calavi, Bénin, 1-199p.

Niyonkuru C. Lalayè PA. 2012. A Comparative Ecological Approach of the Length-Weight Relationships and Condition Factor of Sarotherodon Melanotheron Rüppell, 1852 and Tilapia guineensis (Bleeker 1862) in Lakes Nokoué and Ahémé (Bénin, West Africa). International Journal of Business, Humanities and Technology, 2(3): 41-50. https://www.semanticscholar.org/paper/ A-Comparative-Ecological-Approachof-the-Length- $\%$ E2\%80\%93-\%2C- 
Niyonkuru/c93a37c39535e28679bcdbe4 c01c2e32fe11235c

Panfili J, Durand JD, Mbow A, Guinand B, Diop K, Kantoussan J, Diaga T, Thiaw OT, Albaret JJ, Lae R. 2004. Influence of salinity on life history traits of the bonga shad Ethmalosa finbriata (Pisces, Clupeidae): Comparison between the Gambia and Saloum estuaries. Marine Ecology Progress Series, 270: 241-257. DOI:

http://dx.doi.org/10.3354/meps270241.

Tenda HDL, Zamba AI, Goma-Tchimbakala J, Mikembi LB, PoatyNgot HF, Mamonekene V. 2020. Effets saisonniers sur les relations poids-longueurs et coefficients de condition pour 16 espèces de poissons de la Lagune Mvassa, basse Guinée, République du Congo. Journal of Animal \& Plant Sciences, 44 (1): 7540 7552.

DOI:

https://doi.org/10.35759/JAnmPlSci.v441.1

Teugels GG, Thys van den Audenaerde DFE, 2003. Cichlidae. In Faune des Poissons d'Eaux Douces et Saumâtres de l'Afrique de l'Ouest (Vol. II), Paugy D, Lévêque C, Teugels GG (eds). Collection Faune et
Flore Tropicales n40. Musée Royale de l'Afrique Centrale: Tervuren; Muséum National d'Histoire Naturelle: Paris (France); Institut de Recherche pour le Développement : Paris (France) ; 520 600.

Viaho C. 2014. Inventaire de la faune ichtyologique du lac Ahémé et ses chenaux et quelques aspects bioécologiques des espèces dominantes". Mémoire de Master, Université d'Abomey-Calavi, Bénin, $94 \mathrm{p}$

Villanueva MCS. 2004. Biodiversité et relations trophiques dans quelques milieux estuariens et lagunaires de l'Afrique de l'Ouest : Adaptations aux ressources environnementales. Thèse de doctorat Sciences Agronomiques, Institut national Polytechnique de Toulouse, 1 194p.

Welcomme RL. 2001. Inland Fisheries, Ecology and Management. FAO and Fishing News Books (vol. 1). Blackwell Science Ltd: Oxford; 358p. DOI : https://www.wiley.com/enar/Inland+Fisheries:+Ecology+and+Man agement-p-9780852382844. 